\title{
Multi-frequency sensor for remote measurement of breath and heartbeat
}

\author{
M. Jelen and E. M. Biebl
}

Fachgebiet Höchstfrequenztechnik, Technische Universität München, Germany

\begin{abstract}
Remote measurement of breath and heartbeat is desirable in many situations. It avoids the discomfort resulting from electrodes applied on the skin for long-term patients or during sports acvtivities. Also, surveillance of high security areas or finding survivors of disasters are interesting applications. Common methods identify the movement of heart and thorax by using the range resolution provided by UWB pulse radar systems. In this paper a low-cost approach is presented, that is based on detection of movement by means of Doppler radar sensors. Combining three sensors working in the ISM bands at $433 \mathrm{MHz}, 2.4 \mathrm{GHz}$ and $24 \mathrm{GHz}$, the presence of persons was reliably detected and the frequency of breath and heartbeat was measured.
\end{abstract}

\section{Introduction}

Heartbeat and respiration are a common attribute of all humans. They have a characteristic form and frequency which, in case of the heartbeat, can't be affected voluntarily.

In technical systems (e.g. in cars) there is often the need to detect the presence of a human. This task can be fullfilled by a wide variety of sensors, such as mechanical switches (e.g. in the seat) or ultrasonic sensors.

Also the frequencys of pulse and respiration are often measured. One obvious field of application is the medical sector, but numerous other applications (e.g. sport/fitness) can be thougt of. Currently the measurements are performed by sensors which are in contact with the person beeing observed. This may be a serious disadvantage in situations were measurements must be taken over a long period of time (e.g. long term patients in hospital) or were physical contact is not possible.

An approach of detecting humans by means of radar, mainly for security applications, is described in Greneker

Correspondence to: E. M. Biebl (biebl@tum.de)
(1997). Bimpas et al. (2003) covers the use of a radar system for the detection of persons trapped in building ruins after an earthquake.

This article describes a multi-frequency-sensor approach for both the reliable detection of persons and the measurement of heartbeat and respiration rate.

\section{Physiological and technical basics}

The human heart is approximately the size of a fist. It is located off-center in the thorax, two-third in the left part, one third in the right. In resting state it beats abt. 70 times per minute. With physical effort the activity can increase up to $200 \mathrm{bpm}$. The heart is covered by several layers, the most important are skin, fat and bone. The thickness of these layers varies from person to person.

A movement detection of the heart by means of radar implies the transmission of electromagnetic waves into the human body as well as reflections at a border. To estimate the amount of reflected energy it is necessary to know the dielectric properties of the different body tissues. A rather complete list can be found in Http://niremf.ifac.cnr.it/tissprop/. For the calculation the well-known equations

$R[d B]=10 \log \left(\frac{\left|\underline{Z_{F 2}}\right|-\left|\underline{Z_{F 1}}\right|}{\left|\underline{Z_{F 1}}\right|+\left|\underline{Z_{F 2}}\right|}\right)^{2}$

and

$T[d B]=10 \log \left(\frac{2\left|\underline{Z_{F 2}}\right|}{\left|\underline{Z_{F 1} \mid}\right|+\left|\underline{Z_{F 2}}\right|}\right)^{2} \frac{\left|\underline{Z_{F 2}}\right|}{\left|\underline{Z_{F 1}}\right|}$

can be used. $\underline{Z}_{F}$ can be calculated from $\mu_{r}$ and $\epsilon_{r}$ using the following equation:

$\underline{Z}_{F}=\sqrt{\frac{\mu_{0} \underline{\mu}_{r}}{\epsilon_{0} \underline{\epsilon}_{r}}}=Z_{0} \sqrt{\frac{\underline{\mu}_{r}}{\underline{\epsilon}_{r}}}=Z_{0} \sqrt{\frac{\mu_{r}\left(1-j \tan \delta_{\mu}\right)}{\epsilon_{r}\left(1-j \tan \delta_{\epsilon}\right)}}$

Published by Copernicus GmbH on behalf of the URSI Landesausschuss in der Bundesrepublik Deutschland e.V. 


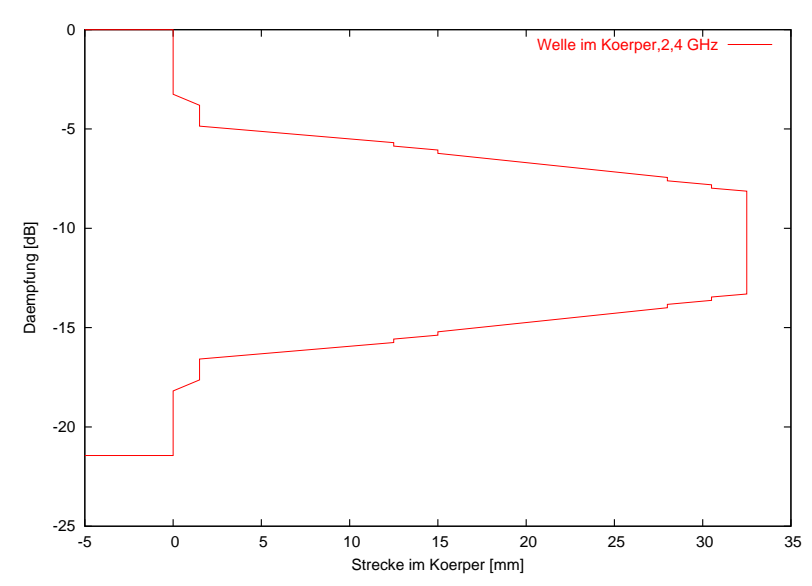

Fig. 1. Propagation of a wave at $440 \mathrm{MHz}$ through the thorax and back.

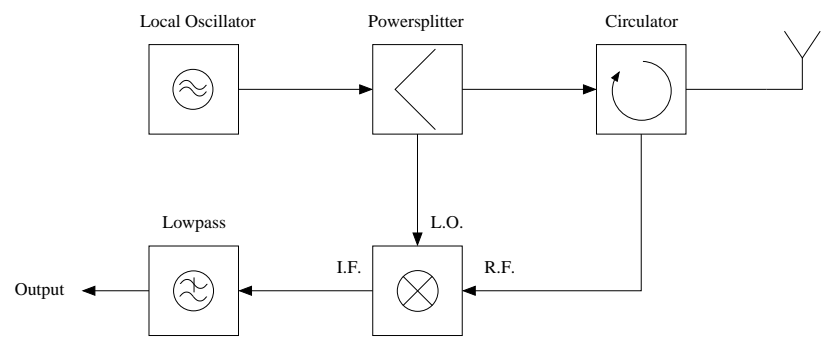

Fig. 2. Simple block diagram of a cw-doppler-radar.

Figure 1 shows an estimation of a wave at $430 \mathrm{MHz}$ being propagated through the body towards the heart and being reflected back on the border fat/heart. The graph shows that a reflected wave is attenuated by approximately $22 \mathrm{~dB}$. The reflection coefficent air-skin is about $2.38 \mathrm{~dB}$ at this frequency. Because the surface of the skin is also much larger than the surface of the heart, the difference between the two reflected signals will be at least $30 \mathrm{~dB}$. Those values are very similiar for $2.4 \mathrm{GHz}$.

The reflection coefficent at the border air/skin at $24 \mathrm{GHz}$ is about $-3.2 \mathrm{~dB}$. In good approximation all power which is transmitted into the body can be considered as lost, so that no reflections from the heart can be measured at this frequency.

Two radar principles could be used for this task: ultrawide-band(UWB)-radar and cw-doppler-radar.

The first one uses short pulses which are reflected at a target. The distance resolution that can be achieved is inverse proportional to the length of the pulses. As the movement of the heart is in the sub-cm-region, very short pulses (and therefore a very high bandwith) would be needed. Despite from frequency regulation issues this would lead to a sophisticated broad-band design, not suitable for a low-cost approach. Staderini covers the use of such a system in medical applications in detail.

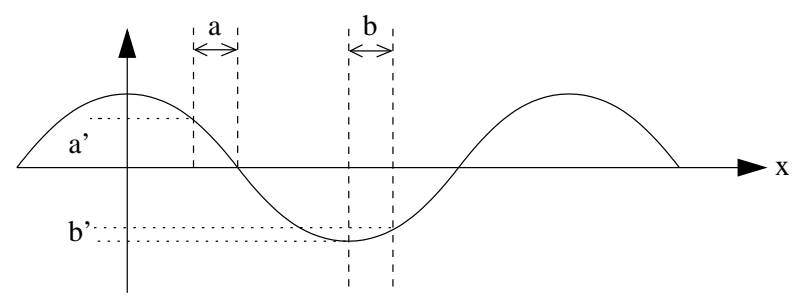

Fig. 3. Influence of phase on mixer output voltage.

The second one is much simpler to design. Figure 2 shows a simple block diagram of a doppler-radar circuit. Contrary to the pulseradar, the signal is sent continously and unmodulated. Therefore only a small bandwith is occupied. The information is contained in amplitude, frequency and phase of the received signal. The signal of the local oscillator is divided in two parts: One part is radiated via the antenna, the second part feeds the LO-port of the receiving mixer. The received signal feeds the IF-port of the mixer. If the frequency of the transmitted signal is $\omega_{T X}$, and the frequency of the received signal is $\omega_{R X}$, and $\varphi_{T X}$ and $\varphi_{R X}$ are the corresponding phases, the following signal can be observed at the IF-port of the mixer:

$$
\begin{aligned}
I F= & A_{1} \cos \left[\left(\omega_{T X}-\omega_{R X}\right) t-\left(\varphi_{T X}-\varphi_{R X)}\right]+\right. \\
& A_{2} \cos \left[\left(\omega_{T X}+\omega_{R X}\right) t-\left(\varphi_{T X}-\varphi_{R X)}\right]+\right. \\
& \cdots
\end{aligned}
$$

$A_{1}$ und $A_{2}$ are constants depending on the mixer. If the frequency of the sent and received signals are equal, the equation simplifies to the following:

$V_{\text {Out }}=A_{1} \cos (\Delta \varphi)$

A dc voltage depending on the phase can be observed at the output of the mixer. If the object which reflects the wave moves around a fixed position (as it is the case with the beating heart) the amplitude of this voltage changes. But the amplitude depends also on the absolute position of the target. This is illustrated in Fig. 3. A phase variation due to a movement within ' $a$ ' causes the voltage change of ' $a$ '. A phase variation about (the same absolut) amount ' $b$ ' on another absolut position causes a much smaller change of ' $b$ '. Because the amplitude of the heartbeat is much smaller than the used wavelengths, this effect is significant for the detection of heartbeats.

To overcome this problem of 'blind spots', the approach shown here uses three sensors at three different frequencies. In almost all cases at least one sensor will be able to achieve a meaningfull measurement result. 


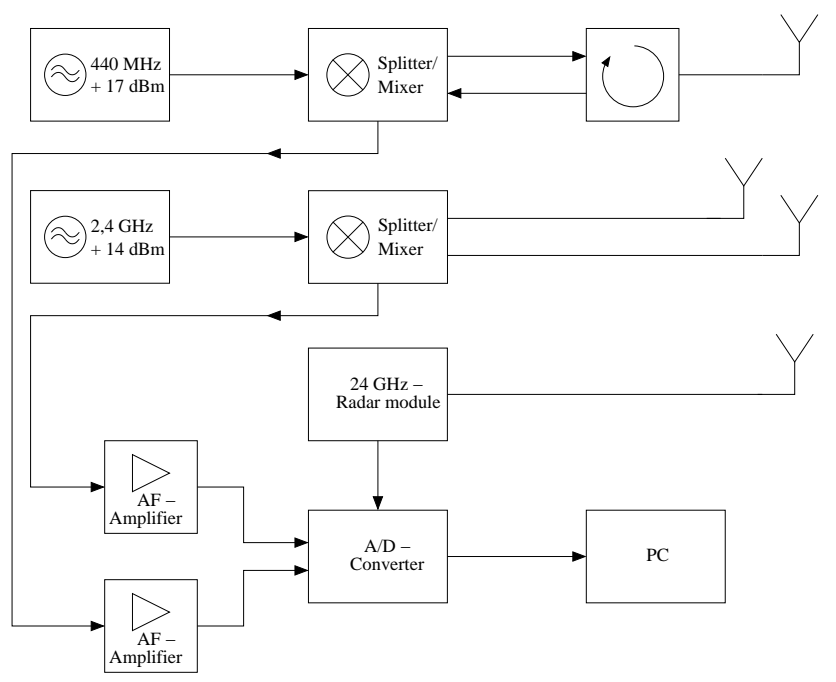

Fig. 4. Block diagram of the system used for the presented measurements.

Table 1. Used frequencies in the ISM range.

\begin{tabular}{lcc}
\hline Wavelength & Frequency range & Output Power \\
\hline $70 \mathrm{~cm}$ & $433,05-434,79 \mathrm{MHz}$ & $0 \mathrm{dBm}$ \\
\hline $12,5 \mathrm{~cm}$ & $2,4-2,5 \mathrm{GHz}$ & $0 \mathrm{dBm}$ \\
\hline $1,25 \mathrm{~cm}$ & $24-24,25 \mathrm{GHz}$ & $0 \mathrm{dBm}$ \\
\hline
\end{tabular}

\section{Measurement setup}

Measurements were performed at three different frequency ranges. To make commercial applications possible, all chosen frequencies are located within the ISM (Industrial, Scientific, Medicine) bands. Table 1 gives an overview over the used frequencies and output powers.

Figure 4 shows a block diagram of the system used for the measurements. For $440 \mathrm{MHz}$, a circulator was available, thus a system according to Fig. 2 could be set up. The RF was supplied by a commercial signal generator. The maximum available output power was $+17 \mathrm{dBm}$. Because a resistive power splitter was used to split the transmit and reference signal and due to the losses in the circulator, approximately $+10 \mathrm{dBm}(10 \mathrm{~mW})$ were available at the antenna.

For $2.4 \mathrm{GHz}$ no circulator was available, so two separate antennas were used for both the transmit and the receive path. Both antennas were realised as patch antennas etched on a low-loss substrate. To keep losses low, the power splitter and mixer were also included on the same substrate. Figure 5 shows the system. The power was also generated by a laboratory generator, and also about $+10 \mathrm{dBm}$ were fed into the transmit antenna.

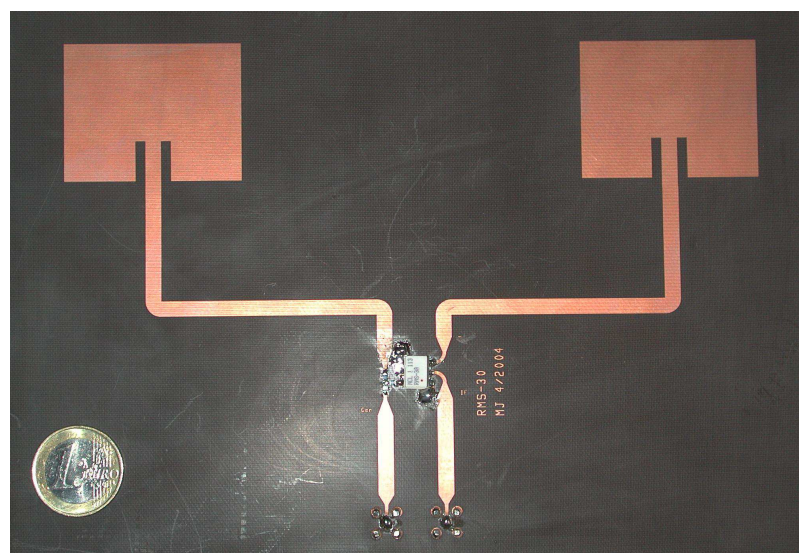

Fig. 5. The sensor used for $2.4 \mathrm{GHz}$.

The $24 \mathrm{GHz}$ measurements were performed using a commercially available doppler radar module with a horn antenna with about $20 \mathrm{dBi}$ antenna gain. The output of this module was ac-coupled, so the response to very slow movements (e.g. breath movement) was strongly attenuated or couldn't be detected at all. This can be seen in the measurement results.

All outputs were fed into a professional 16 bit A/D converter card connected to a notebook. The captured data was processed using MATLAB. First, the dc-offset was removed from the signal by calculating the mean value and subtracting it from all samples. Then the captured time domain data was windowed by a hanning-window to reduce the leakage-effect and converted to the frequency domain using the fast-fourier-transformation (FFT). There the signal was band-pass-filtered with a passband from $0.2 \mathrm{~Hz}$ to $5 \mathrm{~Hz}$. For the time domain representation the filtered signal was transformed back into the time domain.

The achievable frequency resolution $\Delta f$ is direct proportional to the length of the captured sequence $N$ :

$$
\Delta f=\frac{f_{s}}{N}
$$

With a constant sample rate this can be converted into the recording time $t_{s}$ :

$$
\Delta f=\frac{1}{t_{s}}
$$

If a resolution of $0.1 \mathrm{~Hz}$ is needed, the capture time must be at least $10 \mathrm{~s}$.

\section{Measurement results}

For the actual measurements a sample rate $f_{s}=8 \mathrm{kHz}$ was used. For the first measurement a person was seated on a chair and asked to sit quiet without moving and hold the 

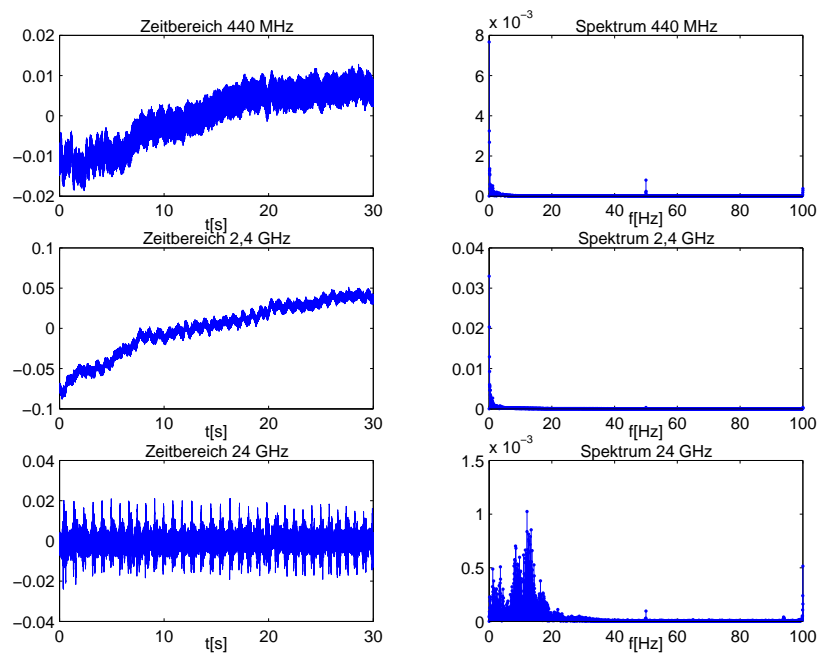

Fig. 6. Raw time- and frequency domain data.
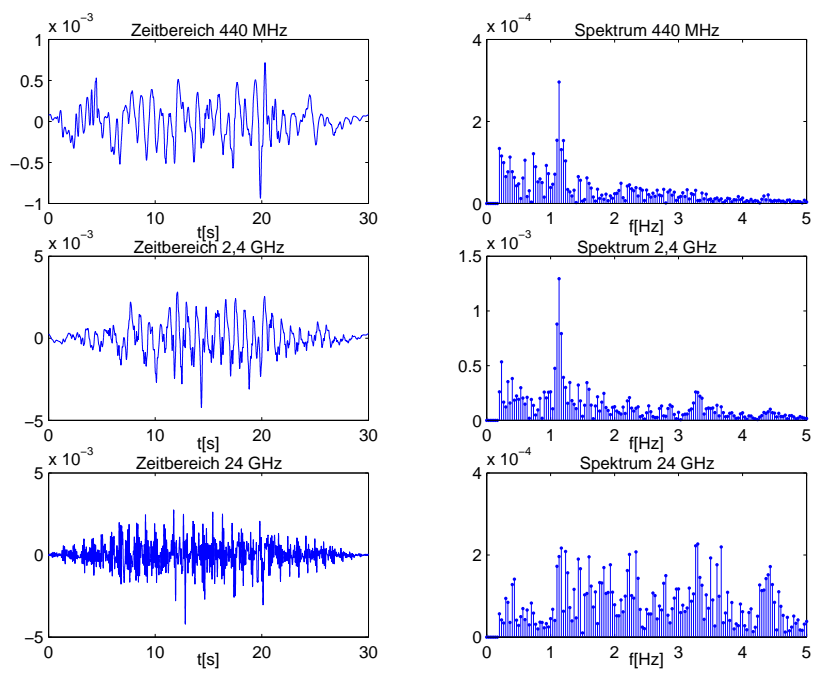

Fig. 7. Filtered time- and frequency domain data.

breath for $30 \mathrm{~s}$. The data from all three sensors were taken parallel. Figure 6 shows the raw time- and frequency domain data as captured from the sensors, Fig. 7 shows the same data after digital filtering.

As one can see in Fig. 6 the spectral components are limited to very low frequencies. Therefore the maximum frequency range in Fig. 7 was reduced to $5 \mathrm{~Hz}$. The frequency resolution with a measurement time of $30 \mathrm{~s}$ is $1 / 30 \mathrm{~Hz}$. A peak is visible at $1.2 \mathrm{~Hz}$. This equals a heart rate of $72 \mathrm{bpm}$ which was confirmed by a parallel 'classical' measurement.

For the second measurement the person was again seated in the chair about $2 \mathrm{~m}$ from the sensors and asked to sit quiet, this time breathing. Figure 8 shows the results. The breath movement is clearly visible in the graphs resulting from the
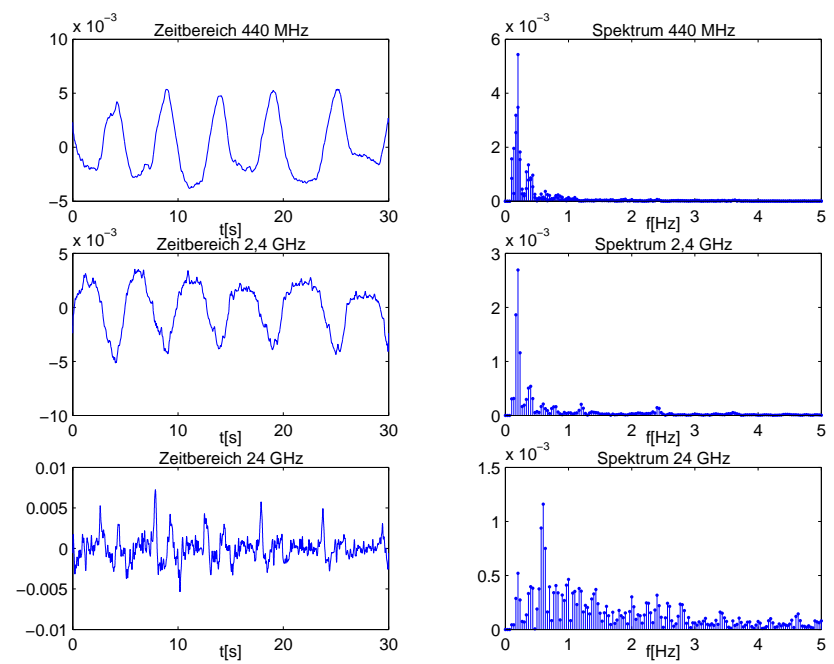

Fig. 8. Breath and heartbeat captured from a distance of $2 \mathrm{~m}$.
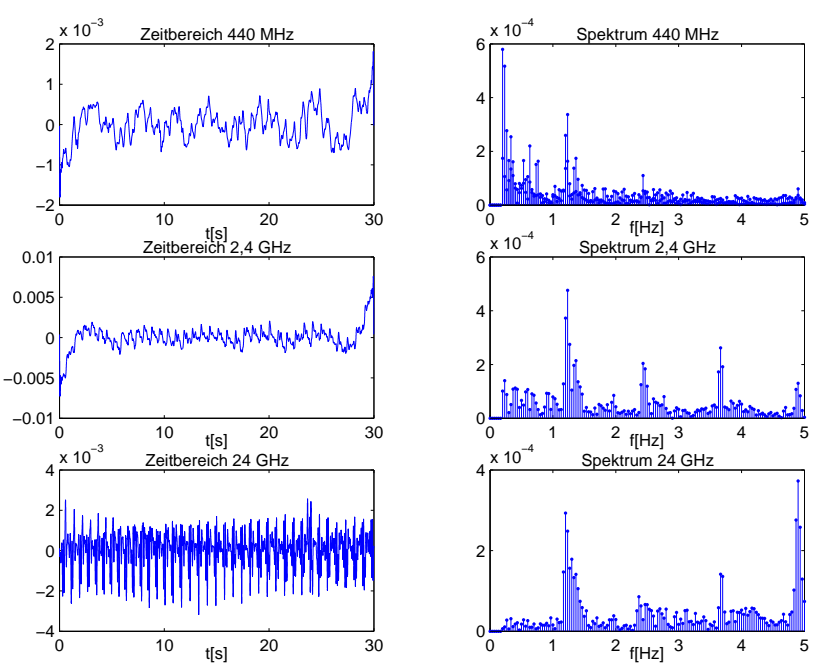

Fig. 9. Heartbeat captured from a distance of $2 \mathrm{~m}$.

measurements at $440 \mathrm{MHz}$ and $2.4 \mathrm{GHz}$. As mentioned before, these low frequencies were cut by the hardware in the $24 \mathrm{GHz}$ sensor. The spectrum has a peak at about $0.26 \mathrm{~Hz}$, which equals a breath frequency of about 15.6 per minute. This was in fact the breath frequeny of the test person.

After the measurement the person was asked to stop breathing so that only the heartbeat could be detected. Figure 9 shows the result. Here a peak in the spectrum is visible at all three measurement frequencies at $1.3 \mathrm{~Hz}$. As a reference, the heart rate of the test person was measured with a pulse watch which showed 79 beats per minute. This equals $1.317 \mathrm{~Hz}$.

Comparing the peak powers of breath and heartbeat with 
Table 2. Increase of maximum compared to reference measurement.

\begin{tabular}{lccc}
\hline Increase & & & \\
\hline Measurement distance & $440 \mathrm{MHz}$ & $2,4 \mathrm{GHz}$ & $24 \mathrm{GHz}$ \\
\hline Distance $1 \mathrm{~m}$ & $>35 \mathrm{~dB}$ & $>35 \mathrm{~dB}$ & $>35 \mathrm{~dB}$ \\
\hline Distance $3 \mathrm{~m}$ & $22,5 \mathrm{~dB}$ & $23,5 \mathrm{~dB}$ & $>40 \mathrm{~dB}$ \\
\hline Distance $3 \mathrm{~m}+$ door & $16,5 \mathrm{~dB}$ & $31,1 \mathrm{~dB}$ & - \\
\hline Distance $8 \mathrm{~m}$ & $21 \mathrm{~dB}$ & $14 \mathrm{~dB}$ & $30 \mathrm{~dB}$ \\
\hline Distance $12 \mathrm{~m}$ & $2,5 \mathrm{~dB}$ & $15,5 \mathrm{~dB}$ & $16,9 \mathrm{~dB}$ \\
\hline
\end{tabular}

the equation

$$
V=20 \log \frac{\text { peak }_{\text {respiration }}}{\text { peak }}
$$

a difference of about $16 \mathrm{~dB}$ for $2.4 \mathrm{GHz}$ and $20 \mathrm{~dB}$ for $440 \mathrm{MHz}$ is found. Assuming that all returning power is reflected at the border air/skin at the breath measurement, these values are close to those predicted before. This may be a hint that actually reflections at the heart were measured.

If only the information is needed whether a person is present within the observed range, a threshold in the power spectrum could be defined. This was tested for different constellations. In all cases the persons were succesfully detected if they were in the line of sight of the antennas. Even through obstacles, such as a closed wooden door, reliable recognition of the presence of persons could be achieved. Table 2 gives an overview over the increase of the low-frequency-spectrum compared to a reference measurement in an empty room.

\section{Conclusions}

CW-doppler-radar sensors at three different frequencies were sucessfully used to detect the presence of persons and to measure heartbeat and respiration rate over a distance of several meters. This was achieved with a low-cost hardware setup which could be easily implemented, for example, in the interior of a car. As expected, $440 \mathrm{MHz}$ and $2.4 \mathrm{GHz}$ show very similiar properties for this application, whereas $24 \mathrm{GHz}$ can only detect body movements due to its very small penetration depth.

The system still has some potential for improvements, for example the sensitivity of the radar sensors could be increased and therefore the output power could be decreased even further. Also only very basic DSP techniques were used for this measurements. A combination of the output from different sensors in combination with more sophisticated DSP algorithms could help to eliminate unwanted responses and overcome the disadvantage of the dependence from the absolute position.

\section{References}

Http://niremf.ifac.cnr.it/tissprop/.

Bimpas, M., Nikellis, K., Paraskevopoulos, N., Economou, D., and Uzunoglu, N.: Devlopement and testing of a detector system for trapped humans in building ruins, in: 33rd European Microwave Conf., 999-1002, 2003.

Greneker, E.: Radar Sensing of Heartbeat and Respiration at a Distance with Security Applications, Proc. of SPIE, 22-27, 1997.

Staderini, E.: UWB Radars in Medicine, IEEE Aerospace and Electronic Systems Magazine, 13-18, 2002. 